\title{
Non-linear EEG Analysis of Idiopathic Hypersomnia
}

\author{
Tarik Al-ani ${ }^{1,2}$, Xavier Drouot ${ }^{3,4}$, and Thi Thuy Trang Huynh ${ }^{3}$ \\ 1 LISV-UVSQ, 10-12 Av. de l'Europe, 78140 Vélizy, France \\ 2 Dept. Informatique, ESIEE-Paris, Cité Descartes-BP 99, 93162 Noisy-Le-Grand, \\ France \\ t.alani@esiee.fr
}

3 APHP, Hôpital Henri Mondor, Service de Physiologie, Centre du Sommeil, 51 av. de Lattre de Tassigny, 94010 Créteil, France

4 EA 4391, Universit Paris 12, France

xavier.drouot@hmn.aphp.fr

\begin{abstract}
The electroencephalogram (EEG) signals are used to analyse and quantify the "depth" of sleep and its dynamic behaviour during night. In this work, we investigate a direct data-driven nonlinear and non-stationary quantitative analysis of sleep $E E G$ issued from patients suffering from idopathic hypersomnia. We show that the minimum weighted average instantaneous frequency appears to be a specific intrinsic characteristic of brain function mechanism in these patients. It could be an interesting new parameter for the quantification of sleep.
\end{abstract}

\section{Introduction}

The hypersomnia can result from a disorder of the central nervous system, what is called primary hypersomnia. There are two main types of primary hypersomnia [123]: narcolepsy and idiopathic hypersomnia $(I H)$. This work concerns only the automatic diagnosis of $I H$. Sleep quality and sleep duration are, in part, governed by a homeostatic process (process $S$ ) which increases during waking and decreases during sleep [4]. This homeostatic drive [5] can be quantified during sleep by calculating the so-called slow wave activity $(S W A)$, with Fast Fourier transform, which is the spectral power of the $0.75-4 \mathrm{~Hz}$ band of the $E E G$ corresponding to the sleep slow waves. For Sleep disorder diagnosis in routine, sleep can be analysed by the study of electroencephalogram (EEG) signals recorded during one night of sleep. Based on $R \mathscr{E} K$ standard rules 6 , these $E E G$ signals are used to quantify the "depth" of sleep and its dynamic behaviour during night. Different interesting computerized (discrete-time) automatic sleep EEG analysis approaches have been developed in the literature [7]. Among these approaches those that are based essentially on some linearity and stationarity hypothesis such as the use of FFT spectrum in short time of a segment of data. However, the accuracy of the FFT calculation is closely related to the choice of the the duration of the signal segment [7]. The main disadvantage of Fourier analysis in signal processing is that it extracts only global features of signals and does

A. Elmoataz et al. (Eds.): ICISP 2010, LNCS 6134, pp. 297-306, 2010.

(C) Springer-Verlag Berlin Heidelberg 2010 
not adapt to analysed signals. Different drawbacks of $F F T$ for $E E G$ analysis are given by Penzel et al. [7.

In the framework of automatic diagnosis of $I H$, the calculation of homeostatic drive during sleep with $S W A$ has been widely used since Borbely's work [4, and is unquestionably a robust parameter to study process $S$ regulation in response to experimental conditions such as sleep deprivation in intra-individual study design. However, the calculation of $S W A$ using FFT has some limitations and caveats that have to be kept in mind. First, as already stated supra, FFT calculation relies on the assumption that sleep $E E G$ is a stationary signal, which is likely not the case. Second, and more importantly, $S W A$ analysis with FFT supposes the choice a priori of the band-width of interest. The lower limit of $S W A$ band has never been strictly defined and varies across studies from $0.25 \mathrm{~Hz}[8$ ] or $0.5 \mathrm{~Hz} 9$ ] to 0.75 [10. However, this could be of physiological importance since the $S W A$ results in the combination of two distinct $E E G$ generators, the cortical slow oscillation (around $0.7 \mathrm{~Hz}$ ) [1] and the thalamic delta clock rhythm (around $2 \mathrm{~Hz}$ ) [12. Such combination is supported by the fact that the dynamics across the night of $S W A$ in response to sleep deprivation, has been showed to be significantly different for the lower part of the $S W A(<1 \mathrm{~Hz})$ and the upper part $(1-4 \mathrm{~Hz})$ in both animals and humans [13 - 14. Thus it is interesting to search for a non linear and non stationary decomposition without a priori choice of frequency bands. In Wavelet Transform (WT) [15] more generalized functions may be used. These functions are characterized by the frequency as well as by the time scales. Although these approaches are more effective than the FFT, they show at the same time much bigger ambiguity in signal decomposition. In this work, we use a more fairly recent technique called the empirical mode decomposition (EMD) [16] for nonlinear and non-stationary time series data analysis such as $E E G$. The $E M D$ behaves like a bank of filters. However, the significant difference with a standard bank of filters is that the modes can be modulated in amplitude and especially in frequency. The advantage of $E M D$ is that it is a data driven approach, i.e. one does not need to define a mother wavelet beforehand. In this work, we are motivated by a direct nonlinear and non-stationary quantitative analysis of sleep $E E G$ issued from healthy subjects and patients suffering from idiopathic hypersomnia based on the instantaneous frequency $(I F)$ using the EMD algorithm. The rest of the paper is organized as follows. Section 2 briefly recalls the principle of the EMD. In section 3 we introduce the $I F$ estimation approaches. Section 4 introduces our methods for hypersomnia analysis. Section [5 is devoted to the results. Finally, section 6 gives some discussion and conclusions on this work.

\section{Empirical Mode Decomposition (EMD)}

The traditional $E M D$, recently introduced by Huang et al. [16, is simply defined by an algorithm based on empirical framework. The EMD can be used to decompose adaptively a signal into a finite number of mono-component signals, which are known as intrinsic mode functions (IMFs) or modes. It considers signals at 
their local oscillations, but they are not necessarily considered in the sens of Fourier harmonics. Their extraction is non-linear, but their recombination for exact reconstruction of the signal is linear. The IMFs admit well-behaved Hilbert transforms (HT) 19 and they satisfy the following properties: they are symmetric, different IMFs yield different instantaneous local frequencies as functions of time that give sharp identifications of embedded structures. The decomposition is done linearly or non-linearly depending on the data. This complete and almost orthogonal decomposition is empirically realised by identifying the physical local characteristic time scales intrinsic to these data, which is the lapse between successive extrema. For more details on the EMD algorithm, see [16.

\section{Instantaneous Frequency Estimation (IF)}

The $I F$ was originally defined in the context of FM modulation theory in communications. Ville [17] unified the work done by Carson and Fry [18] and Gabor [19] and noted that since the $I F$ was time-varying, there should intuitively be some instantaneous spectrum associated with it-with the mean value of the frequencies in this instantaneous spectrum being the IF [20]. The importance of the IF concept stems from the fact that in many applications, like our $E E G$ signal analysis, spectral characteristics (in particular the frequency of the spectral peaks) are varying with time, i.e. nonstationary. In this case, the IF is an important characteristic; it is a time-varying parameter which defines the location of the signals spectral peak as it varies with time. Conceptually it may be interpreted as the frequency of a sine wave which locally fits the signal under analysis. Physically, it has meaning only for monocomponent signals, where there is only one frequency or a narrow range of frequencies varying as a function of time 20]. The $I F$ has been applied with success to EEG signal analysis [21. The EMD method is not itself a time-frequency representation. The use of the concept of $I F$ is here interesting since the (IMFs) are mono-component signals. This implies that there is a single frequency at any time. There are several techniques to estimate the IF using the derivative of the phase of a signal. Hilbert-Huang transform (HHT) method 22] is an empirically based data-analysis method based on EMD and Hilbert transform HT [19] which is the most common method to estimate the $I F$. Its basis of expansion is adaptive, so that it can produce physically meaningful representations of data from nonlinear and non-stationary processes. . The results indicate that both these two methods can abstract the main characters of the EEG. But HHT can more accurately express EEG distribution in time and frequency domain. Thats because it can produce a self-adaptive basis according to the signal and obtain local and instantaneous frequency of EEG. However, the determination of this instantaneous quantity is based on the integration of the signal, which is a global operation on signal duration with the same weight for the past and for the future. Moreover, the definition of this instantaneous quantity is based on a non-causal and non-instantaneous filter and on the FT of the analytical signal. The estimation of instantaneous quantities requires the values of the signal that are not yet observed. Thus, we can not do that before 
that the signal has been fully observed. To avoid this constraint, there are other approaches privileging essentially the notion of instantaneity to estimate the $I F$ and the $I A$ of a signal. These approaches use only the amplitudes of the signal and, if necessary, its derivative at the given instant 23 .

\subsection{Teager-Kaiser Energy Operator (TKEO)}

Kaiser 23 proposed a very simple and fast algorithm to estimate the energy. That operator is called Teager-Kaiser Energy Operator (TKEO), provided that the restriction related to the signal bandwidth (narrow-band signal) is respected. In the following, the discrete version of this algorithm is presented.

Consider a discrete real value signal $x(n)$. The discrete TKEO, $\Psi_{d}[x(n)]$, may be written as $\Psi_{d}[x(n)]=x^{2}(n)-x(n-1) x(n+1)$. This operator requires no assumption on the stationarity of the signal. Its formulation shows that it is local and easy to implement. Indeed, only three samples are required to calculate the energy at each moment. The estimation of the $I F$ and $I A$ of a discrete signal $x(n)$ sampled at a frequency $f_{e}$ is inspired by the discrete-time energy separation algorithm - 1 noted $\left(D E S A_{1}\right)$ developed by Maragos et al. [24]-26]

$$
\begin{aligned}
& |a(n)|=\sqrt{\frac{\Psi_{d}[x(n)]}{1-\left(1-\frac{\Psi_{d}[y(n)]+\Psi_{d}[y(n+1)]}{4 \Psi_{d}[x(n)]}\right)^{2}}}, \\
& \Omega_{i}(n)=\arccos \left(1-\frac{\Psi_{d}[y(n)]+\Psi_{d}[y(n+1)]}{4 \Psi_{d}[x(n)]}\right), f_{i}(n)=\frac{\Omega_{i}(n)}{2 \pi} f_{e},
\end{aligned}
$$

with $y(n)=x(n)-x(n-1)$. Finally, the $I F$ is estimated by Potamianos et al. 27] have concluded on the interest of this operator for the estimation of the IF for demodulation in speech compared to the approach based on the analytical signal.

\section{Methods}

\subsection{EEG Records}

$E E G$-records obtained from healthy females as well as females suffering from $I H$ were collected by the team at "Sleep Laboratory, Department of Physiology Functional Explorations at Henri Mondor Hospital (Créteil-France)" for about 8 hours of sleep. There are two types of collected EEG-records. The first records were obtained from healthy volunteers: 9 healthy females aged 25 - 35 years while the second records were obtained from age-matched females that suffering from $I H$ diagnosed with polysymptomatic form of $I H: 11$ females aged 25 - 35 years. Diagnosis of $I H$ were established using the International criteria [5]. All the EEG signals, issued from the placement of electrodes C4 - O2 according to the International 10-20 system, were sampled at $200 \mathrm{~Hz}$, with 16 -bit resolution. The studied EEG signals were scored with 30 -second epochs based on R\&K standard criteria. The hypnograms corresponding to these signals were also used in our work. 


\subsection{Application of EMD to our EEG records}

In our work, the EMD algorithm is used to calculate the IMFs or modes. Five modes were generated. Then, the instantaneous frequencies (IFs) were calculated using Equations (1] 2). Analysing all night sleep necessitates analysing each of the modes on all night. To do that, the EMD is applied on each epoch (30 seconds) of the $E E G$ signal to retrieve the mode of interest. For each epoch of the calculated mode, we calculate the mean of $I F$ and the energy of the $E E G$ signal of the epoch. Finally, we calculate and Plot the $I F$ and the energy as a function of the time. To reduce noise, the values are smoothed using median filter for the energy and Savitzky and Golay filter [28, for the IF. This will provide the evolution of $I F$ and energy during all night sleep.

\section{Results}

We started by applying first the EMD algorithm on one epoch (30 seconds) of the $E E G$ signal corresponding to a healthy female "ab". In our analysis, each mode reveals predominantly a certain range of frequency and that the frequency of mode 1 is nearly two times greater than that of mode 2 and so on.

\subsection{Identification of the Descriptive Parameter of Slow Deep Sleep by the Study of 9 Healthy Females}

The IFs of the different modes during a night's sleep were calculated. We observe that the $I F$ of stage 4 is always lower than the other stages. Besides, the evolution of the IFs during the night is almost the same for all 5 modes, only the amplitude of the variations decreases more and more from mode 1 to mode 5 . This led us to calculate the weighted average instantaneous frequency (WAIF) 29] which has a linear relationship to the individual instantaneous frequencies. This weighting will take into account the amplitudes of the different modes.

Let $x(t)$ a signal decomposed by the EMD algorithm to $N$ modes. The WAIF at time $t$ is given by

$$
W A I F(t)=\frac{\sum_{n=1}^{N} a_{n}^{2}(t) f_{n}(t)}{\sum_{n=1}^{N} a_{n}^{2}(t)},
$$

where $a_{n}(t)$ and $f_{n}(t)$ are the $I A$ and the $I F$ at time $t$ respectively. The weighted average instantaneous frequency (WAIF) of one epoch is the average of the weighted averages instantaneous frequency (WAIFs) of all its points. For the nine healthy females, we noted that, whatever the stage, the WAIF is lower during sleep than in awakening state and it reveals very well the slow deep sleep (stages 3 and 4), particularly the very deep sleep (stage 4). Indeed, during stage 4, the WAIF decreases significantly. In addition, the stage-4 periods are well localised in terms of time and duration. A statistical analysis using repeated analysis of variance (ANOVA) showed a significant effect of sleep stages (wake, stage 1, stage 2 to 4 , and REM) on WAIF for all sleep epochs $(\mathrm{F}=62.5$ and $\mathrm{p}<0.0001)$. Post hoc Fisher test also 

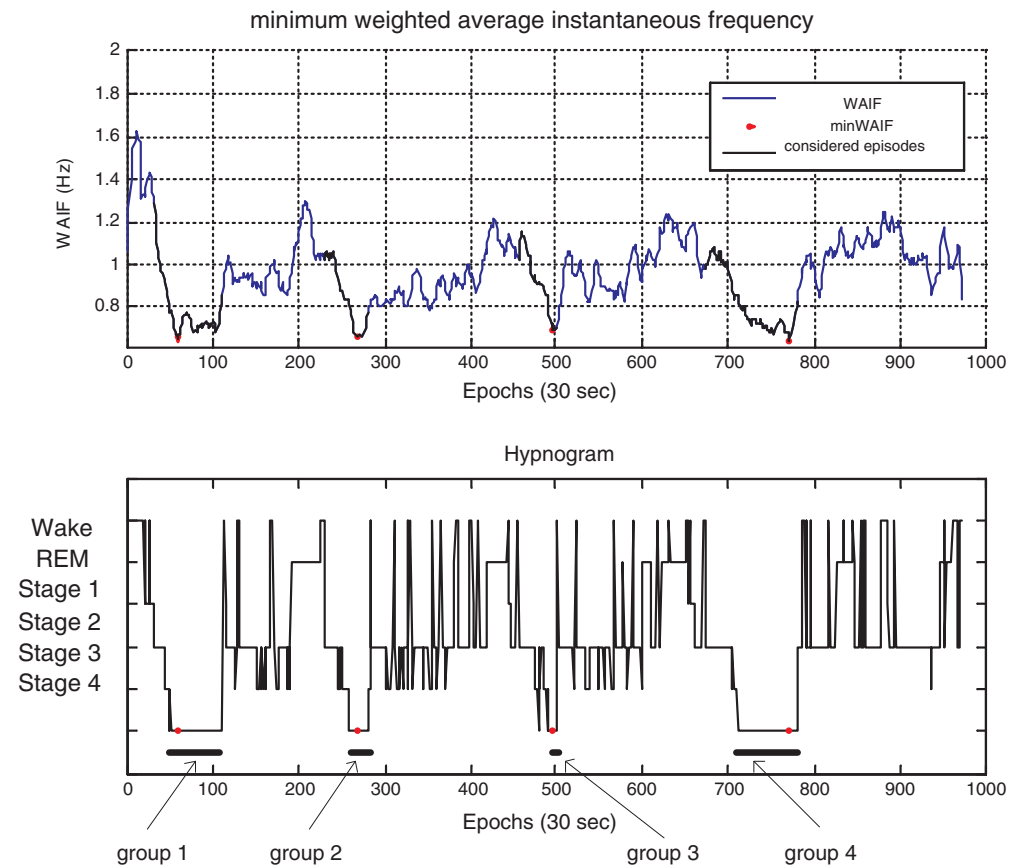

Fig. 1. The min WAIF of the episodes of deep sleep for the healthy female "ab". The different min WAIF are indicated by red dots.

showed that WAIF in each sleep stage significantly differs from the three others ( $\mathrm{p}<0.006$ for all pairs). We noticed that the most interesting parameter could be the minimum weighted average instantaneous frequency ( min WAIF) reached during deep sleep episodes (one episode is a series of 30-second epochs). These episodes of deep sleep contain inevitably stage 4 (or stage 3 in case of a new type of scoring in which stages 3 and 4 are grouped) and possibly stages 2 and 3 . Hence, we developed a simple algorithm to obtain min WAIF of deep sleep episodes. Fig. 11 shows that the min WAIF could be considered as a new parameter for quantification of sleep EEG.

\subsection{Comparison of the minWAIF for the Two Female Groups}

We compared the min WAIF for the two female groups (9 healthy females and 11 females suffering from $I H)$. We realize two types of In this section, we compare the min WAIF for the two female groups (9 healthy females and 11 females suffering from $I H)$. We realize two types of interpretation: visual interpretation and statistical interpretation.

Visual interpretation. In Fig. 2, one can already see that the mean of the min WAIF value in healthy females never drops below $0.6 \mathrm{~Hz}$, while that of females suffering from $I H$ drops as low as $0.45 \mathrm{~Hz}$. In addition, the range of the 


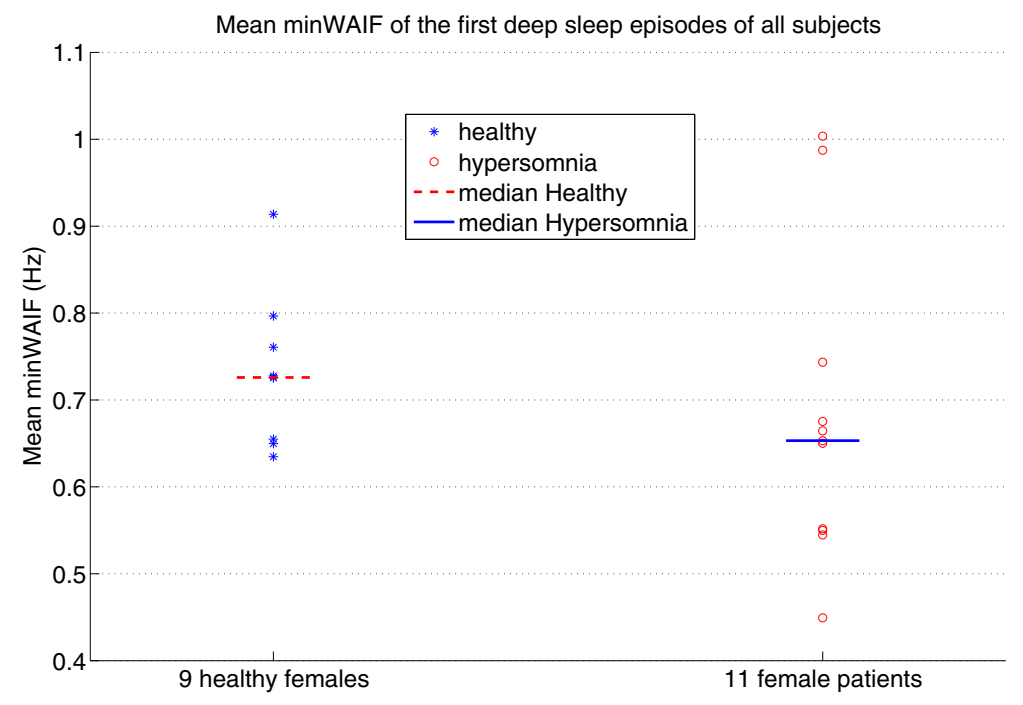

Fig. 2. The mean of the min WAIF of the first four deep sleep episodes of 2 groups

mean of min WAIF values in healthy females is more narrow than that of female patients.

Statistical interpretation. For all tests, the level of significance, $\alpha$, was set to $5 \%$. In order to find possible differences between healthy females and females suffering from $I H$, we compared the $\min W A I F$ values in both groups (9 healthy females vs. 11 female patients using the Mann-Whitney nonparametric test. The test result shows that the $\min W A I F$ value was significantly lower in patients than in healthy females $0.7172 ;[0.6787-0.7635]$ (median; 25 th percentile -75 th percentile) for the 9 healthy females vs. 0.6524 ; [0.5640-0.7761] for the 11 female patients $(\mathrm{p}<0.05$, Mann-Whitney test).

\section{Conclusions}

In this work, we studied a new approach for nonlinear and non-stationary quantitative analysis of sleep $E E G$ signals issued from healthy females as well as females suffering from idiopathic hypersomnia. This approach is based on the empirical mode decomposition (EMD) algorithm and the instantaneous frequency (IF) of the intrinsic mode functions (IMFs). The IF, which is generated by the Teager-Kaiser energy operator (TKEO), is motivated by the objective of seeking a relevant descriptive parameter of the neurological hypersomnia. Our study has been validated on sleep EEG data issued from healthy femals and patient females suffering from $I H$. Because of the similarity in the evolution of the $(I F)$ in the five relevant modes, the weighted average instantaneous frequency (WAIF) was then calculated by weighting with the amplitudes of the different 
modes. The results showed that the WAIF reveals well deep sleep (especially stage 4). However, it should be noted that the physiological interpretation of these quantities is difficult and delicate because their values do not represent in themselves the "true" physiological frequencies that are normally observed. We are interested only by their variation over time since it reflects the evolution of physiological frequencies of brain waves. Unlike the $I F$, the evolution of the signal energy is more discriminating with only modes 2,3 and 4 . The reduction of energy at the end of the night can be explained by the fact that in general the amount of deep sleep gradually decreases during a night sleep. A physiological explanation could be attributed to the decrease in frequency and increase in energy during deep sleep. The deeper the sleep is, the more brain activities slow down and become synchronised, resulting in the slower and bigger brain waves observed during deep sleep. Finally, we decided to go further by examining the minimum weighted average instantaneous frequency (min WAIF) reached during deep sleep episodes. Statistical tests showed that min WAIF is stable during the night and significantly lower among patients suffering from $I H$ than among healthy females. The min WAIF appears to be a specific intrinsic characteristic of brain function mechanism in patients suffering from $I H$. It could be an interesting new parameter for the quantification of sleep $E E G$. Most of patients have a min WAIF in the range of the healthy subjects. However, the three patients who have a min WAIF clearly below the lowest healthy's min WAIF also suffer from severe difficulties to awake in the morning. Further studies will be necessary to determine if this low WAIF is specific to these awakening difficulties.

The results of the analysis of the min WAIF seem interesting and promising. However, it remains to verify in a future study the reproducibility of this parameter from one night to another in order to study the first night effect. This study needs $E E G$ records of two consecutive nights for the same healthy females. In order to obtain a more statistically significant results, several $E E G$ records issued from a sufficient number of healthy subjects as well as patients suffering from $I H$ will also be required.

\section{Acknowledgments}

The authors would like to thank CEPHALON for the financial support (indemnity) through Thi Thuy Trang Huynh (first author) research scholarship.

\section{References}

1. Furukawax, T.: Heinrich Bruno Schindler's description of narcolepsy in 1829. Neurology 37, 146 (1987)

2. Roth, B.: Narcolepsy and hypesomnia: review and classification of 642 personally observed cases. Schweiz Arch Neurol Neurochir Psychiatr 199(1), 31-41 (1975)

3. Kirsch, D.B., Chervin, R.D.: Idiopathic Hypersomnia and Reccurent Hypersomnia,plus 0.5em minus 0.4emSleep Disorders And Neurologic Diseases, ch. 9, 2nd edn. Antonio Culebras, New York (2007) 
4. Borbely, A.A.: A two process model of sleep regulation. Hum. Neurobiol. 1, 195-204 (1982)

5. American Academy of Sleep Medicine. In: ICSD-The International Classification of Sleep Disorders, 2nd edn. (2005)

6. Rechtschaffen, A., Kales, A.: A Manual of Standardized Terminology, Techniques and Scoring System for Sleep Stages of Human Subjects,plus 0.5em minus 0.4em, Brain Inform. Service/Brain Res. Inst., Univ. California, Los Angeles (1968)

7. Penzel, T., Conradt, R.: Computer based sleep recording and analysis. Sleep Medicine Reviews 4(2), 131-148 (2000)

8. Guilleminault, C., Kirisoglu, C., da Rosa, A.C., Lopes, C., Chan, A.: Sleepwalking, a disorder of NREM sleep instability. Sleep Medicine 7(2), 163-170 (2006)

9. Preud'homme, X.A., Lanquart, J.P., Krystal, A.D., Bogaerts, P., Linkowsk, P.: Modeling slow-wave activity dynamics: does an exponentially dampened periodic function really fit a single night of normal human sleep? Clin. Neurophysiol. 119, 2753-2761 (2008)

10. Sforza, E., Gaudreau, H., Petit, D., Montplaisir, J.: Homeostatic sleep regulation in patients with idiopathic hypersomnia. Clinical Neurophysiology 111, 277-282 (2000)

11. Achermann, P., Borbely, A.A.: Low-frequency $(<1 \mathrm{~Hz})$ oscillations in the human sleep electroencephalogram. Neuroscience 81, 213-222 (1997)

12. Amzica, F., Steriade, M.: Electrophysiological correlates of sleep delta waves. Electroencephalogr. Clin. Neurophysiol. 107, 69-83 (1998)

13. Vyazovskiy, V.V., Achermann, P., Tobler, I.: Sleep homeostasis in the rat in the light and dark period. Brain Res. Bull. 74, 37-44 (2007)

14. Campbell, G., Higgins, L.M., Darchia, N., Feinberg, I.: Homeostatic behavior of fast Fourier transform power in very low frequency non-rapid eye movement human electroencephalogram. Neuroscience 140, 1395-1399 (2006)

15. Mallat, S.G.: A theory for multiresolution signal decomposition: the wavelet representation. IEEE Transaction on Pattern Analysis and Machine Intelligence 11(7), 674-693 (1989)

16. Huang, N.E., Shen, Z., Long, S.R., Wu, M.C., Shih, H.H., Zheng, Q., Yen, N.C., Tung, C.C., Liu, H.H.: The empirical mode decomposition and the Hilbert spectrum for nonlinear and non-stationary time series analysis. Proc. of the Royal Society 454, 903-995 (1998)

17. Ville, J.: Theorie et application de la notion de signal analytic. Cables et Transmissions 2A(1), 61-74 (1948); Translation by I. Selin, Theory and applications of the notion of complex signal, Report T-92, RAND Corporation, Santa Monica, CA

18. Carson, J., Fry, T.: Variable frequency electric circuit theory with application to the theory of frequency modulation. Bell System Tech. J. 16, 513-540 (1937)

19. Gabor, D.: Theory of communication. Proc. IEE 93(III), 429-457 (1946)

20. Boashash, B.: Estimating and interpreting the instantaneous frequency of a signalPart 1. Proc. IEEE 80, 520-538 (1992)

21. Ktonas, P.Y., Papp, N.: Instantaneous envelope and phase extraction from real signals: Theory, implementation and an application to EEG analysis. Signal Processing 2, 373-385 (1980)

22. Huang, N.E., Shen, Z., Long, S.R.: A new review of nonlinear water waves: The Hilbert spectrum. Annu. Rev. Fluid Mech. 31, 417-457 (1999)

23. Kaiser, J.F.: On a simple algorithm to calculate the 'energy' of a signal. In: Proc. of IEEE ICASSP 1990, Albuquerque, New Mexico, Avril 1990, pp. 381-384 (1990) 
24. Maragos, P., Kaiser, J.F., Quatieri, T.F.: On separating amplitude from frequency modulations using energy operators. In: Proc. of IEEE ICASSP 90, vol. 2, pp. 1-4 (1992)

25. Maragos, P., Kaiser, J.F., Quatieri, T.F.: Energy separation in signal modulations with application to speech analysis. IEEE Trans. on Signal Processing 41(10), 3024-3051 (1993)

26. Maragos, P., Kaiser, J.F., Quatieri, T.F.: On amplitude and frequency demodulation using energy operators. IEEE Trans. on Signal Processing 41(4), 1532-1550 (1993)

27. Potamianos, A., Maragos, P.: A comparison of the energy operator and Hilbert transform approach to signal and speech demodulation. Sig. Proc. 37, 95-120 (1994)

28. Savitzky, A., Golay, M.J.E.: Smoothing and Differentiation of Data by Simplified Least Squares Procedures. Analytical Chemistry 36(8), 1627-1639 (1964)

29. Loughlin, P.J.: The time-dependent weighted average instantaneous frequency. In: Proc. IEEE Intl. Symp. Time-Frequency and Time-Scale Analysis, October 1998, pp. 97-100 (1998) 